\title{
Mastofauna de médio e grande porte na RPPN da UNISC, RS, Brasil
}

\author{
Edson Fiedler de Abreu Júnior ${ }^{1,2}$ \& Andreas Köhler ${ }^{1}$ \\ ${ }^{1}$ Departamento de Biologia e Farmácia, Universidade de Santa Cruz do Sul - UNISC \\ Av. Independência, 2293, Bairro Universitário, CEP 96815-900, \\ Santa Cruz do Sul, RS, Brasil, www.unisc.br \\ ${ }^{2}$ Autor para correspondência: Edson Fiedlerde Abreu Júnior, e-mail: edabreujr@hotmail.com
}

ABREU JR, E.F. \& KÖHLER, A. Mammalian fauna of medium and large sized in the RPPN of UNISC, RS, Brazil. Biota neotrop., 9(4): http://www.biotaneotropica.org.br/v9n4/en/abstract?inventory+bn02109042009.

\begin{abstract}
The aim of this study was to conduct an inventory on the mammal fauna present at UNISC's private natural reserve (RPPN), in order to better contribute with data on the occurrence and distribution of mid and large-sized mammal species at Rio Grande do Sul's Serra Geral region. Field samplings were conducted from November 2008 to April 2009. Additional previously collected data were also used. Methods used were live visual searching and camera-traps. Records of 16 mammal species were obtained, belonging to 12 families and six Orders: two Didelphimorphia, one Xenarthra, two Primates, six Carnivora, one Artiodactyla and four Rodentia. Two other species were not recorded according to our survey protocols and, consequently, were not considered in the analysis of occurrence consistency and spatial occupation. Six species were considered as occasional, seven were accessory, and only one, Cerdocyon thous, was constant. Nine species (64\%) were considered as specialists regarding the habitats they occupied, and five (36\%) were generalists. Diet categories analysis indicated seven omnivorous species (43.75\%), four frugivorous (25\%), three carnivores (18.75\%) and two herbivorous $(12.5 \%)$. Among the 16 species recorded, seven are classified as under any level of threat of extinction for the State of Rio Grande do Sul, one is nationally threatened, and two other are considered globally "near threatened". The fact that $44 \%$ of the species are experiencing any level of extinction threat reinforces the importance of the RPPN for the conservation of the regional mammal fauna.
\end{abstract}

Keywords: mammals, Serra Geral, inventory, tracks, camera-traps.

ABREU JR, E.F. \& KÖHLER, A. Mastofauna de médio e grande porte na RPPN da UNISC, RS, Brasil. Biota neotrop., 9(4): http://www.biotaneotropica.org.br/v9n4/pt/abstract?inventory+bn02109042009.

Resumo: Buscou-se com este trabalho inventariar a mastofauna da RPPN da UNISC, contribuindo com dados a respeito da ocorrência e distribuição de espécies de mamíferos de médio e grande porte na Região da Serra Geral do Rio Grande do Sul. As amostragens de campo realizaram-se nos meses de novembro de 2008 a abril de 2009, porém, foram acrescentados registros tomados anteriormente. Utilizou-se os métodos de busca visual e de armadilhas fotográficas. Foram obtidos registros de 16 espécies de mamíferos silvestres, pertencentes a 12 famílias e seis ordens: dois Didelphimorphia, um Xenarthra, dois Primates, seis Carnivora, um Artiodactyla e quatro Rodentia. Duas espécies não foram registradas nas amostragens padronizadas, conseqüentemente não submetidas às análises de constância de ocorrência e ocupação espacial. O índice de constância de ocorrência revelou que seis espécies foram consideradas ocasionais, sete acessórias e apenas uma constante, Cerdocyon thous. A análise da ocupação espacial das espécies demonstrou que nove espécies (64\%) são especialistas, quanto ao habitat ocupado, e cinco $(36 \%)$ generalistas. A análise das categorias de dieta evidenciou que sete espécies são onívoras (43,75\%), quatro frugívoras (25\%), três carnívoras (18,75\%) e duas herbívoras $(12,5 \%)$. Do total das 16 espécies silvestres, sete aparecem em algum nível de ameaça de extinção no Rio Grande do Sul, uma encontra-se ameaçada nacionalmente e duas estão definidas como "quase ameaçada" em âmbito mundial. O enquadramento de $44 \%$ das espécies em alguma categoria de ameaça de extinção enaltece a importância da RPPN para a preservação da mastofauna regional.

Palavras-chave: mamíferos, Serra Geral, inventário, pegadas, armadilhas fotográficas. 


\section{Introdução}

A mastofauna desempenha papel fundamental na manutenção do equilíbrio dos ecossistemas, envolvendo-se nos mais distintos processos ecológicos, entre eles, o controle populacional de suas presas e a constante regeneração das matas. Conforme Tonhasca Jr (2005) inúmeras espécies vegetais dependem dela para a dispersão de suas sementes. Algumas espécies são indicadoras ambientais, refletindo a preservação do local onde ocorrem (Mazzolli 2006). A perda e a fragmentação de habitat, resultantes de atividades humanas, constituem as maiores ameaças aos mamíferos terrestres no Brasil, sendo que os mamíferos de grande e médio porte sofrem ainda a pressão de caça, prática ilegal no país há mais de 35 anos (Costa et al. 2005).

A região central do Estado conta com um histórico de destruição e degradação dos ambientes naturais desde a chegada dos primeiros imigrantes, restando, hoje, poucos fragmentos florestais capazes de suportar populações viáveis de mamíferos de médio e grande porte, que necessitam de grandes áreas para cumprir suas funções vitais. No Vale do Rio Pardo a fragmentação dos habitats está intimamente relacionada com a cultura do tabaco, já que o mesmo caracteriza-se por ser a principal região fumicultora do Brasil (Campos \& Delevatti 2003). Conforme o Conselho Regional de Desenvolvimento do Vale do Rio Pardo (COREDE 1998), um dos problemas ambientais da região é o desmatamento para ampliação de solo agrícola e aproveitamento de lenha para os fornos de secagem de fumo.

Neste cenário, as unidades de conservação são uma alternativa para garantir a preservação da diversidade biológica. Entretanto, a intromissão de espécies exóticas e/ou domésticas nas áreas de reservas biológicas afetam diretamente as espécies silvestres, podendo levá-las a extinção (Primack 1998). Dentre os animais domésticos o cão interage com espécies nativas através da predação, competição por recursos limitados e introdução de doenças, ocasionando sérios danos a fauna silvestre (Rodrigues 2002, Gaspar 2005).

Os pré-requisitos indispensáveis para o desenvolvimento de ações conservacionistas, principalmente no que se refere à sua biodiversidade, estão na dependência do conhecimento básico das espécies e sua distribuição espacial, onde se inclui os inventários mastofaunísticos (Kasper et al. 2007a). Cerqueira (2001) ressalta que a grande maioria das áreas de preservação não conta sequer com inventários que determinem parâmetros de biodiversidade, destacando a importância dos trabalhos de levantamento de dados básicos. Desta forma, buscou-se com este trabalho inventariar a mastofauna da Reserva Particular do Patrimônio Natural (RPPN) da Universidade de Santa Cruz do Sul (UNISC), contribuindo com dados a respeito da ocorrência e distribuição de espécies de mamíferos de médio e grande porte na Região da Serra Geral do Rio Grande do Sul.

\section{Material e Métodos}

\section{1. Área de estudo}

A RPPN da UNISC foi criada através da portaria no ${ }^{\circ} 15$ de 18 de Março de 2009, possuindo uma área de 221,39 hectares. Localizase no município de Sinimbu, no Vale do Rio Pardo, Rio Grande do Sul (Figura 1). Está situada nas coordenadas geográficas $29^{\circ} 23^{\prime} \mathrm{S}$ e $52^{\circ} 32^{\prime} \mathrm{O}$ e limita-se a oeste com o Rio Pardinho. De acordo com IBGE (1986) a RPPN insere-se na unidade geomorfológica Serra Geral, mais especificadamente, na Área Serrana.

A área de estudo compõe-se de um relevo acidentado esculpido pela erosão regressiva do Rio Pardinho e seus tributários (Ab’Saber 1969). A altimetria da área apresenta grande variação hipsométrica, desde 150 m, no leito do Rio Pardinho, até 650 m, na porção leste da Reserva. A região apresenta um clima subtropical, correspondente ao clima Cfa II2b de Köppen. A temperatura média do mês mais

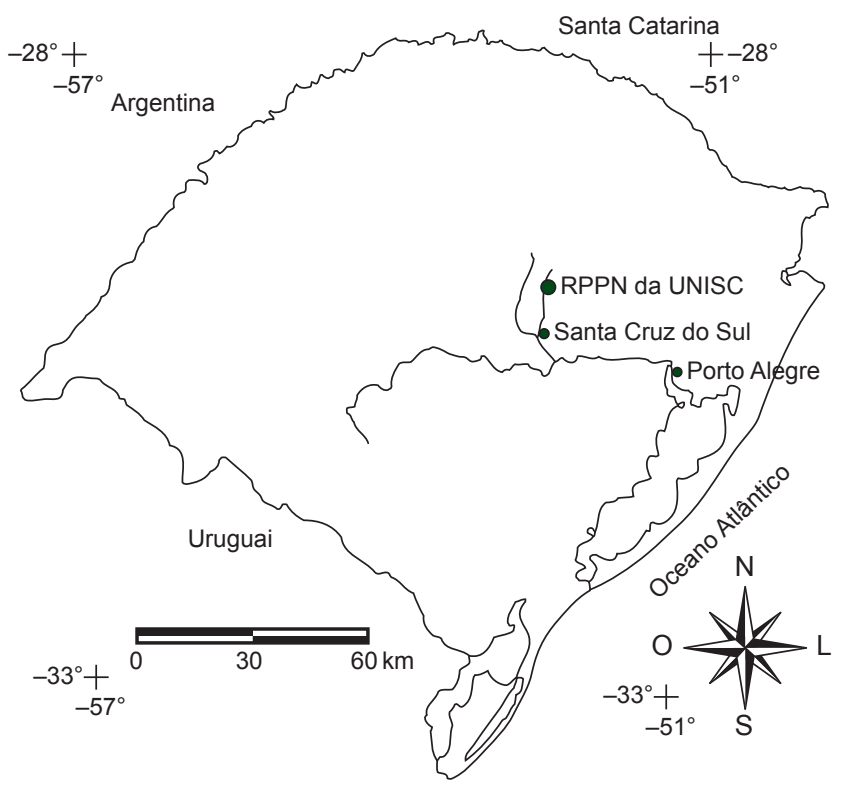

Figura 1. Mapa de localização da RPPN da UNISC no município de Sinimbu, Rio Grande do Sul, Brasil.

Figure 1. Location of UNISC's RPPN at Sinimbu Municipaly, Rio Grande do Sul, Brazil.

quente é superior a $22^{\circ} \mathrm{C}$ e a do mês mais frio superior a $3{ }^{\circ} \mathrm{C}$, com temperatura média anual superior a $18{ }^{\circ} \mathrm{C}$ (UNISC, 2005).

A formação florestal local é a Floresta Estacional Decidual, integrante do Domínio Mata Atlântica (Marcuzzo et al. 1998). Porém, a existência de componentes florísticos da Floresta Ombrófila Mista, como a Araucaria angustifolia (Bertol.) Kuntze, demonstram uma situação de transição de formações florestais na região. A RPPN da UNISC constitui-se, em geral, de uma área de vegetação secundária em variados estágios de regeneração, possuindo fragmentos de mata com inúmeras trilhas para o deslocamento humano e com presença de espécies vegetais exóticas (p. ex. Hovenia dulcis Thunb, Citrus limon (L.) Burm. e Tecoma stans (L.) ex. Kunth).

\section{Amostragem}

As amostragens de campo realizaram-se nos meses de novembro de 2008 a abril de 2009. A permanência mensal na área de estudo foi de, em média, quatro dias. Para complementação do levantamento foram considerados registros fotográficos de rastros e de visualizações, tomados pelos autores em visitas ocasionais a área de estudo antes do início deste trabalho, nos anos de 2007 e 2008.

O principal método utilizado para a coleta dos dados foi a busca visual, considerando os registros diretos e indiretos da presença de espécies de mamíferos de médio e grande porte (Santos et al. 2008). A amostragem direta correspondeu às visualizações e a amostragem indireta os vestígios, como, fezes, pêlos, tocas, marcas em árvores, odor, restos mortais e rastros.

A busca visual deu-se percorrendo trilhas e áreas fora das trilhas na Reserva. Foram realizadas a pé em horários variados, desde o amanhecer até a noite, e com duração variada. Obteve-se, também, registros visuais percorrendo a estrada que dá acesso a sede da Reserva com veículo motorizado, em velocidade aproximada de $30 \mathrm{Km} / \mathrm{h}$, sempre à noite (Kasper et al. 2007b).

Para cada registro direto ou indireto foram tomadas as coordenadas geográficas do local e anotadas, além destas, a data, o horário, o 
tipo de registro e, nos avistamentos, o número de indivíduos. Todos os registros indiretos e alguns diretos foram fotografados.

Complementar ao método de busca visual aplicou-se o método de armadilhas fotográficas, utilizando um equipamento fotográfico confeccionado e uma armadilha fotográfica digital modelo Wildview Xtreme 2.0. O esforço amostral total correspondeu a 109 armadilhasnoite.

Para a identificação das espécies foram consultados Canevari \& Vaccaro (2007), Borges \& Tomás (2004), Oliveira \& Cassaro (1999), Becker \& Dalponte (1991) e Langguth \& Anderson (1980). A nomenclatura adotada e a ordem de apresentação das espécies estão conforme Wilson \& Reeder (2005).

\section{Análise dos dados}

Os registros resultantes da busca visual e das armadilhas fotográficas foram somados para o processamento dos dados e, para este, excluiu-se as espécies detectadas anteriormente ao inicio das amostragens padronizadas (Leopardus wiedii e Sphiggurus villosus). Submeteu-se os resultados ao estimador de riqueza de espécies Jackknife, no programa PC-ORD ver. 4 (Mccune \& Mefford 1999).

A constância de ocorrência das espécies foi avaliada pelo índice de Silveira-Neto et al. (1976), utilizando a escala de Santos et al. (2008), onde as espécies encontradas em mais de 50\% das amostras (campanhas mensais de amostragem) são consideradas constantes; aquelas presentes entre 25 e $50 \%$ são acessórias e aquelas encontradas em menos de $25 \%$ das amostras são consideradas ocasionais. Analisou-se a ocupação espacial das espécies (Santos et al. 2008) nos diversos ambientes disponíveis (margens de cursos-d'água, mata e área antropizada com vegetação de capoeira), sendo consideradas especialistas aquelas cuja frequiência de ocorrência foi igual ou superior a $70 \%$ em um dos ambientes estudados, e generalista aquelas cuja freqüência de ocorrência foi inferior a $70 \%$.

As espécies ocorrentes foram classificadas quanto à dieta seguindo Reis et al. (2006) e conforme o risco de extinção no Livro vermelho da Fauna Ameaçada de Extinção no Rio Grande do Sul (Fontana et al. 2003), no Livro Vermelho da Fauna Brasileira Ameaçada de Extinção (Machado et al. 2008) e pela Lista Vermelha das Espécies Ameaçadas (IUCN 2008).

\section{Resultados e Discussão}

Foram obtidos registros de 16 espécies de mamíferos silvestres de médio e grande porte, pertencentes a 12 famílias e seis ordens: dois Didelphimorphia, um Xenarthra, dois Primates, seis Carnivora, um Artiodactyla e quatro Rodentia (Tabela 1). Com base no Índice de Jackknife deveriam ocorrer de 19 a 20 espécies na RPPN da UNISC. Carnivora correspondeu à ordem com maior riqueza, confirmando estudos realizados na Mata Atlântica (p. ex. Gaspar 2005, Kasper et al. 2007b, Negrão \& Valladares-Pádua 2006). Mesmo não amostrando mamíferos de pequeno porte, a segunda ordem com maior número de espécies encontradas foi Rodentia, resultado que demonstra a grande diversidade desta ordem, que constitui-se em uma das mais diversas mundialmente (Reis et al. 2006).

O número de espécies de mamíferos de médio e grande porte registradas iguala-se ao registrado para o Planalto Meridional (Cerveira 2005), onde também foram detectadas 16 espécies. Entretanto este número revela-se inferior em comparação a outros trabalhos, por exemplo: Gaspar (2005) registrou 23 espécies em um fragmento de floresta atlântica semidecídua no Estado de São Paulo; Kasper et al. (2007a) registraram, para o Vale do Taquari, 26 espécies de mamíferos de médio e grande porte; Kasper et al. (2007b) catalogaram a ocorrência de 29 espécies, para o Parque Estadual do Turvo.
Além das espécies silvestres, também foram registradas quatro espécies domesticas: Canis familiaris Linnaeus, 1758 (cachorrodoméstico), Sus scrofa Linnaeus, 1758 (porco-doméstico), Capra hircus Linnaeus, 1758 (cabra) e Bos tauros Linnaeus 1758 (boi). Estas espécies percorrem a área da Reserva devido à existência de propriedades rurais junto aos seus limites. Dentro da área da RPPN da UNISC é constante a visualização de cães circulando livremente e, por duas ocasiões, deparou-se com indivíduos do gênero Mazama em fuga destes animais.

Das 16 espécies, 14 foram detectadas através da busca visual e das armadilhas fotográficas nos seis meses de amostragens, porém, duas espécies tiveram seus registros somente em período anterior ao início das amostragens padronizadas: Leopardus wiedii e Sphiggurus villosus. A aplicação do índice de constância de ocorrência revelou que das 14 espécies, seis foram consideradas ocasionais, sete acessórias e, apenas uma, constante. Destaca-se, no entanto, que das seis espécies ocasionais, cinco (Dasypus novemcinctus, Cebus nigritus, Alouatta guariba clamitans, Eira barbara e Nasua nasua) apresentam a mesma área de ocupação, a mata, ambiente onde a disponibilidade de registros indiretos é reduzido, portanto a ocasionidade destas espécies deve ser interpretada com cautela. Para quatro das espécies acessórias percebe-se uma relação de ocupação de margens de cursosd'água (Chironectes minimus, Lontra longicaudis, Cuniculus paca e Hydrochoerus hydrochaeris), ambientes comuns na área de estudo, não somente pela presença do Rio Pardinho, mas também pela existência de pequenos córregos. A grande disponibilidade destes locais torna-se determinante para a ocorrência destas espécies. A única espécie classificada como constante foi Cerdocyon thous, caracterizada pela alta plasticidade e tolerância a ambientes antropizados (Fonseca et al. 1996, Reis et al. 2006).

A análise da ocupação espacial das espécies na área de estudo demonstrou que nove espécies (64\%) são especialistas, quanto ao habitat ocupado, e cinco (36\%) generalistas, resultado similar ao encontrado por Santos et al. (2008), onde 57\% das espécies registradas foram consideradas habitat-especialistas. As espécies especialistas, Chironectes minimus, Lontra longicaudis, Nectomys squamipes, Cuniculus paca e Hydrochoerus hydrochaeris, têm como habitat margens ou leito de cursos-d'água, sendo restritas a estes ambientes (Silva 1994, Borges \& Tomás 2004, Reis et al. 2005, Reis et al. 2006). Cebus nigritus, Alouatta guariba clamitans e Eira barbara, utilizam como habitats exclusivamente as matas, apenas Dasypus novemcinctus pode ocupar outras formações vegetais (Silva 1994, Borges \& Tomás 2004, Reis et al. 2005, Reis et al. 2006). Quanto as espécies generalistas Didelphis albiventris, Cerdocyon thous, Nasua nasua e Procyon cancrivorus há possibilidade de ocupação de áreas antropizadas, pois apresentam tolerância e diversificação nos ambientes ocupados (Silva 1994, Borges \& Tomás 2004, Reis et al. 2005, Reis et al. 2006).

A análise das categorias de dieta revelou que sete espécies são onívoras $(43,75 \%)$, quatro frugívoras $(25 \%)$, três carnívoras $(18,75 \%)$ e duas herbívoras (12,5\%), a predominância dos onívoros corrobora com o trabalho de Negrão \& Valladares-Pádua (2006). Deve-se, no entanto, destacar que o grupo dos carnívoros está representado por três espécies, destas, duas têm hábitos semi-aquáticos e somente uma predadora terrestre. Refletindo a baixa densidade de potenciais presas no meio terrestre na vegetação predominantemente secundária da área de estudo.

Com relação aos níveis de ameaça das espécies registradas sete aparecem em alguma categoria de ameaça de extinção no Rio Grande do Sul, uma encontra-se ameaçada nacionalmente e duas estão definidas como "quase ameaçada" a nível mundial. Assim, 44\% das espécies registradas na área de estudo estão sofrendo algum tipo de pressão e sua ocorrência pode estar comprometida. 
Tabela 1. Listagem taxonômica (ordem, família e espécie), forma de registro, categoria de dieta, índice de constância de ocorrência e ocupação espacial dos mamíferos encontrados na RPPN da UNISC, Sinimbu, Rio Grande do Sul. Forma de registro: visualização (V), pegadas (P), restos mortais (RM), fezes (F), vocalização (VO), registro fotográfico (RF). Categoria de dieta: onívoro (Oni), frugívoro (Fru), carnívoro (Car) e herbívoro (Her). Índice de constância (IC): acessória (A), ocasional (O) e constante (C). Ocupação espacial: margens de cursos-d'água (MC), mata (MA) e área antropizada (AA).

* Registros anteriores aos seis meses de amostragens.

Table 1. Taxonomic listing (order, family and species), type of record, categories of diet, constancy of occurrence index and spatial occupation of mammals at the RPPN of UNISC, Sinimbu, Rio Grande do Sul. Type of record: visualization (V), tracks (P), remains (RM), faeces (F), vocalization (VO) and photography record (RF). Categories of diet: omnivorous (Oni), frugivorous (Fru), carnivorous (Car) and herbivorous (Her). Constancy index (IC): accessory (A), occasional (O) and constant (C). Spatial occupation: edge of water bodies (MC), forest (MA) and anthropogenic disturbance area (AA).

* Records prior to six months of sampling.

\begin{tabular}{|c|c|c|c|c|c|c|}
\hline \multirow[t]{2}{*}{ Táxon } & \multirow{2}{*}{$\begin{array}{c}\text { Forma de } \\
\text { Registro }\end{array}$} & \multirow{2}{*}{$\begin{array}{c}\text { Categoria } \\
\text { de dieta }\end{array}$} & \multirow[t]{2}{*}{ IC } & \multicolumn{3}{|c|}{ Ocupação espacial (\%) } \\
\hline & & & & MC & MA & $\mathbf{A A}$ \\
\hline \multicolumn{7}{|l|}{ Didelphimorphia } \\
\hline \multicolumn{7}{|l|}{ DIDELPHIDAE } \\
\hline Chironectes minimus (Zimmermann, 1780) & $\mathrm{P}$ & Car & A & 100 & - & - \\
\hline Didelphis albiventris Lund, 1840 & $\mathrm{RM}$ & Oni & A & - & 50 & 50 \\
\hline \multicolumn{7}{|l|}{ Xenarthra } \\
\hline \multicolumn{7}{|l|}{ DASYPODIDAE } \\
\hline Dasypus novemcinctus Linnaeus, 1758 & $\mathrm{P}^{*}, \mathrm{RM}$ & Oni & $\mathrm{O}$ & - & 100 & - \\
\hline \multicolumn{7}{|l|}{ Primates } \\
\hline \multicolumn{7}{|l|}{ CEBIDAE } \\
\hline Cebus nigritus (Goldfuss, 1809) & $\mathrm{V}$ & Oni & $\mathrm{O}$ & - & 100 & - \\
\hline \multicolumn{7}{|l|}{ ATELIDAE } \\
\hline Alouatta guariba clamitans Cabrera, 1940 & $\mathrm{VO}$ & Her & $\mathrm{O}$ & - & 100 & - \\
\hline \multicolumn{7}{|l|}{ Carnivora } \\
\hline \multicolumn{7}{|l|}{ FELIDAE } \\
\hline Leopardus wiedii (Schinz, 1821) & $\mathrm{P} *$ & Car & - & - & - & - \\
\hline \multicolumn{7}{|l|}{ CANIDAE } \\
\hline Cerdocyon thous (Linnaeus, 1766) & $\mathrm{V}, \mathrm{P}, \mathrm{RF}^{*}$ & Oni & $\mathrm{C}$ & 9 & 64 & 27 \\
\hline \multicolumn{7}{|l|}{ MUSTELIDAE } \\
\hline Lontra longicaudis (Olfers, 1818) & $\mathrm{V}^{*}, \mathrm{P}, \mathrm{F}$ & Car & A & 100 & - & - \\
\hline Eira barbara (Linnaeus, 1758) & V & Oni & $\mathrm{O}$ & - & 100 & 1 \\
\hline \multicolumn{7}{|l|}{ PROCYONIDAE } \\
\hline Nasua nasua (Linnaeus, 1766) & $\mathrm{V}, \mathrm{P}, \mathrm{RM}$ & Oni & $\mathrm{O}$ & - & 50 & 50 \\
\hline Procyon cancrivorus (Cuvier, 1798) & $\mathrm{P}$ & Fru/Oni & A & 40 & - & 60 \\
\hline \multicolumn{7}{|l|}{ Artiodactyla } \\
\hline \multicolumn{7}{|l|}{ CERVIDAE } \\
\hline Mazama sp. Rafinesque, 1817 & $\mathrm{~V}, \mathrm{P}$ & Fru/Her & A & - & 33 & 67 \\
\hline \multicolumn{7}{|l|}{ Rodentia } \\
\hline \multicolumn{7}{|l|}{ CRICETIDAE } \\
\hline Nectomys squamipes (Brants, 1827) & $\mathrm{P}$ & Oni & $\mathrm{O}$ & 100 & - & - \\
\hline \multicolumn{7}{|l|}{ CUNICULIDAE } \\
\hline Cuniculus paca (Linnaeus, 1758) & $\mathrm{P}, \mathrm{RF}$ & Fru/Her & A & 73 & 27 & - \\
\hline \multicolumn{7}{|l|}{ CAVIIDAE } \\
\hline Hydrochoerus hydrochaeris (Linnaeus, 1766) & $\mathrm{P}, \mathrm{F}$ & Her & A & 75 & 25 & - \\
\hline \multicolumn{7}{|l|}{ ERETHIZONTIDAE } \\
\hline Sphiggurus villosus (Cuvier, 1823) & $\mathrm{V}^{*}$ & Fru/Her & - & - & - & - \\
\hline
\end{tabular}

As ameaças às espécies de primatas relacionam-se diretamente com suas dependências a habitats florestais, Aloutta guariba clamitans está classificada com "Vulnerável" no Estado e Cebus nigritus aparece como "Dados insuficientes" no Estado e como "Quase ameaçada" mundialmente. Segundo Silva (1994), os bugios são os primatas mais comuns no Rio Grande do Sul, porém, as populações de Aloutta guariba clamitans do Planalto das Araucárias estão entre as que sofrem maior pressão antrópica (Marques 2003). Na área da RPPN da UNISC o avistamento de bandos de Cebus nigritus é mais freqüente que de bandos de Aloutta guariba clamitans, em algumas ocasiões foi possível a contagem de mais de 10 indivíduos em um bando de Cebus nigritus.

Eira barbara, Nasua nasua e Leopardus wiedii são espécies, também, associadas a florestas e necessitam da disponibilidade 
e boa qualidade desses ambientes. Dentre estas espécies, Nasua nasua, classificada como "Vulnerável" no Rio Grande do Sul, foi a que apresentou o maior número de detecções, tendo relatos de moradores locais da presença de grupos com cerca de 15 indivíduos. Eira barbara, classificada como "Vulnerável" no Estado, e Leopardus wiedii, classificado como "Vulnerável" no Rio Grande do Sul e no Brasil e "Quase ameaçada" em nível mundial, tiveram um único registro cada, o que sugere que um empenho maior seja realizado a fim de obter-se mais dados da ocorrência destas espécies, que carecem de uma avaliação mais acurada de suas populações.

Duas espécies, Chironectes minimus e Lontra longicaudis, classificadas como "Vulnerável" no âmbito Estadual são adaptadas a vida aquática. Suas ameaças estão intimamente ligadas à poluição das águas e suas margens, destruição da vegetação ripária e alta densidade populacional humana (Fonseca et al. 1994). Na área de estudo ocorrem na porção leste, curso do Rio Pardinho, sendo Lontra longicaudis mais freqüentemente registrada, por suas fezes e seu odor marcante.

A espécie mais ameaçada, no Rio Grande do Sul, registrada neste trabalho é Cuniculus paca, que encontra-se na categoria "em perigo". Conforme Christoff (2003), a destruição e descaracterização do habitat, combinados à caça predatória, representam as maiores ameaças à espécie. $\mathrm{Na}$ área de estudo tem sido amplamente registrada por pegadas às margens do Rio Pardinho e por registros fotográficos. Sendo esta uma espécie cinegética ressalta-se seu registro, pois, apesar da RPPN da UNISC ser uma área cercada por propriedades rurais e de fácil transito humano, esta espécie pode ter encontrado nela um refúgio para sua sobrevivência.

As demais espécies, hoje, não aparecem em categorias de extinção, o que não indica que não estejam sofrendo com as ações antrópicas de degradação e fragmentação de seus habitats, mas que apresentam uma plasticidade e tolerância maior a estes efeitos, além de serem de ampla distribuição (Santos et al. 2008).

\section{Considerações Finais}

Registrou-se 16 espécies de mamíferos de médio e grande porte na RPPN da UNISC, inferindo a ocorrência destas para a Área Serrana da unidade geomorfológica Serra Geral. A ausência de determinadas espécies com distribuição local histórica e a não constância da maioria das espécies registradas, pode estar vinculada ao passado de degradação e destruição sofrido pela região. Desde a chegada dos imigrantes, as matas vêm dando lugar às lavouras e os animais silvestres vêm perdendo espaço para as criações domésticas, além de serem abatidos pela caça ilegal. Soma-se a estes fatos a pequena extensão da Reserva e suas características paisagísticas, apresentando áreas com vegetação em estágio inicial de regeneração.

Porém, o enquadramento de $44 \%$ das espécies em alguma categoria de ameaça de extinção, enaltece a importância da RPPN para a preservação da mastofauna regional. A ocorrência de Cuniculus paca, Chironectes minimus, Lontra longicaudis, Leopardus wiedii, entre outras, evidencia a existência atual de populações de importantes espécies de mamíferos em locais deficientes de registros no Rio Grande do Sul. Estes dados vêm, portanto, aumentar a área de distribuição destas espécies e colaborar no preenchimento de lacunas do conhecimento mastofaunístico.

Faz-se necessário, no entanto, ressaltar a presença de animais não silvestres na área da Reserva. Frente a esta situação, medidas coerentes e efetivas de controle de animais domésticos devem ser tomadas a fim de assegurar a sobrevivência e a viabilidade de populações da fauna silvestre local, em especial da mastofauna. Estas medidas devem partir de um simples diálogo informativo com os moradores lindeiros e estender-se a uma fiscalização eficiente. Para uma unidade de con- servação cumprir com seus propósitos é indispensável à colaboração da população de entorno, principalmente em Reservas pequenas, onde as interferências antrópicas vizinhas são mais acentuadas.

\section{Agradecimentos}

Agradecemos ao Fábio Dias Mazim pelas sugestões e auxilio nas identificações. Ao Felipe Bortolotto Peters pelo auxilio nas identificações. Ao Eduardo Ribeiro Machado pelo auxilio no desenvolvimento do equipamento fotográfico. Ao Samuel Lopes Oliveira e Rafael Barbizan Sühs pela disponibilização de fotografias de registros e ajuda nas atividades de campo. Ao Paulo Francisco Kuester, Alexandre Somavilla, Humberto Mohr, Fabiane Noronha e Tatiana Straatman pela ajuda nas atividades de campo.

\section{Referências}

AB'SÁBER, A.N. 1969. Um conceito de geomorfologia a serviço das pesquisas sobre o Quaternário. Bol. Geomorfologia. (18):1-23.

BECKER, M. \& DALPONTE. J.C. 1991. Rastros de mamíferos silvestres brasileiros: um guia de campo. Editora da Universidade de Brasília, Brasília, $180 \mathrm{p}$.

BORGES, P.A.L. \& TOMÁS, W.M. 2004. Guia de rastros e outros vestígios de mamíferos do pantanal. Embrapa Pantanal, Corumbá, 148 p.

CAMPOS, H.A. \& DELEVATTI, D.M. 2003. Caracterização ambiental da região do Vale do Rio Pardo. In Agenda 21 regional do Vale do Rio Pardo (RS) (H. A. Campos \& L. C. Schceider, eds). EDUNISC, Santa Cruz do Sul, p. 11-54.

CANEVARI, M. \& VACCARO, O. 2007. Guía de mamíferos del sur de América del Sur. 1 ed. LOLA, Buenos Aires, 424 p.

CERQUEIRA, R. 2001. Um sistema de monitoramento e inventário da biodiversidade terrestre do Brasil. In Conservação e biodiversidade em ecossistemas tropicais: avanços conceituais e revisão de novas metodologias de avaliação e monitoramento (I. Garay \& B. F. S. Dias, eds). Vozes, Petrópolis, p. 147-149.

CERVEIRA, J. 2005. Mamíferos silvestres de médio e grande porte no Planalto Meridional: suas relações com a fragmentação da paisagem e a presença do gado. Dissertação de Mestrado, Universidade Federal do Rio Grande do Sul, Porto Alegre.

CHRISTOFF, A.U. 2003. Roedores e Lagomorfos. In Livro vermelho da fauna ameaçada de extinção no Rio Grande do Sul (C.S. Fontana, G.A. Bencke \& R.E. Reis, eds). EDIPUCRS, Porto Alegre, 632 p.

Conselho Regional de Desenvolvimento - COREDE. 1998. Plano estratégico de desenvolvimento do Vale do Rio Pardo. EDUNISC, Santa Cruz do Sul.

COSTA, L.P., LEITE, Y.R.L., MENDES, S.L. \& DITCHFIELD, A.D. 2005 Conservação de mamíferos no Brasil. Megadiversidade. 1(1):103-112.

FONSECA, G.A.B., HERRMANN, G., LEITE, Y.R.L., MITTERMEIER, A.B.R. \& PATTON, J.L. 1996. Lista anotada dos mamíferos do Brasil. Occasional Papers in Conservation Biology, Washington, p. 1-38.

FONSECA, G.A.B., RYLANDS, A.B., COSTA, C.M.R., MACHADO, R.B. \& LEITE, Y.R.L. 1994. Livro vermelho dos mamíferos brasileiros ameaçados de extinção. Fundação Biodiversitas, Belo Horizonte, 459 p.

FONTANA, C.S., BENCKE, G.A. \& REIS, R.E. 2003. Livro vermelho da fauna ameaçada de extinção do Rio Grande do Sul. EDIPUCRS, Porto Alegre, $632 \mathrm{p}$.

GASPAR, D.A. 2005. Comunidade de mamíferos não-voadores de um fragmento de floresta atlântica semidecídua do município de Campinas, SP. Tese de Doutorado, Universidade Estadual de Campinas, Campinas.

Instituto Brasileiro de Geografia e Estatística - IBGE. 1986. Levantamento de recursos naturais. IBGE, Rio de Janeiro. (Projeto Radam Brasil, v. 33).

International Union for Conservation of Nature and Natural Resources - IUCN. 2008. The IUCN Red List of Threatened Species. IUCN, Cambridge. http://www.iucnredlist.org/ (último acesso em 02/06/2009) 
KASPER, C.B., FELDENS, M.J., MAZIN, F.D., SCHNEIDER, A., CADEMARTORI, C.V. \& GRILLO, H.C.Z. 2007a. Mamíferos do Vale do Taquari, Região Central do Rio Grande do Sul. Biociências. 15(1):53-62.

KASPER, C.B., MAZIM, F.D., SOARES, J.B.G., OLIVEIRA, T.G. \& FABIÁN, M.E. 2007b. Composição e abundância relativa dos mamíferos de médio e grande porte no Parque Estadual do Turvo, Rio Grande do Sul, Brasil. Rev. Bras. Zool. 24(4):1087-1100.

LANGGUTH, A. \& ANDERSON, S. 1980. Manual de identificación de los mamíferos del Uruguay. Universidad de la República, Montevideo.

MACHADO, A.M.B., DRUMMOND, G.M., PAGLIA, A.P. 2008. Livro vermelho da fauna brasileira ameaçada de extinção. 1 ed. MMA; Fundação Biodiversitas, Brasília, 1420 p.

MARCUZZO, S., PAGEL, S.M. \& CHIAPPETTI, M.I.S. 1998. A reserva da biosfera da Mata Atlântica no Rio Grande do Sul: situação atual, ações e perspectivas. Conselho Nacional da Reserva da Biosfera da Mata Atlântica, São Paulo.

MARQUES, A.A.B. 2003. Primatas. In Livro vermelho da fauna ameaçada de extinção no Rio Grande do Sul (C.S. Fontana, G.A. Bencke \& R.E. Reis, eds). EDIPUCRS, Porto Alegre, 632 p.

MAZZOLLI, M. 2006. Persistência e riqueza de mamíferos focais em sistemas agropecuários no planalto meridional brasileiro. Tese de Doutorado, Universidade Federal do Rio Grande do Sul, Porto Alegre.

MCCUNE, B. \& MEFFORD, M.J. 1999. Multivariate analysis of ecological data. MjM Softwere Desing, Gleneden Beach.

NEGRÃO, M.F.F. \& VALLADARES-PÁDUA, C. 2006. Registros de mamíferos de maior porte na Reserva Florestal do Morro Grande, São Paulo. Biota Neotrop. 6(2): http://www.biotaneotropica.org.br/v6n2/pt/ abstract?article+bn00506022006 (último acesso em 09/08/2009)

OLIVEIRA, T.G. \& CASSARO, K. 1999. Guia de identificação dos felinos brasileiros. Sociedade de Zoológicos do Brasil, São Paulo, 60 p.
PRIMACK, R.B. 1998. Essentials of conservation biology. 2 ed. Sunauer Associates, Sunderland, 564 p.

REIS, N.R., PERACCHI, A.L., FANDIÑO-MARIÑO, H. \& ROCHA, V.J. 2005. Mamíferos da Fazenda Monte Alegre, Paraná. Eduel, Londrina, $177 \mathrm{p}$.

REIS, N.R., PERACCHI, A.L., PEDRO, W.A. \& LIMA, I.P. 2006. Mamíferos do Brasil. Nélio R. Reis, Londrina, 437 p.

RODRIGUES, F.H.G. 2002. Ecologia do lobo guará na estação Ecológica de Águas Emendadas, DF. Tese de Doutorado, Universidade de Campinas, Campinas.

SANTOS, T.G., SPIES, M.R., KOPP, K., TREVISAN, R. \& CECHIN, S.Z. 2008. Mamíferos do campus da Universidade Federal de Santa Maria, Rio Grande do Sul, Brasil. Biota Neotrop. 8(1): http://www.biotaneotropica. org.br/v8n1/pt/abstract?inventory+bn00508012008 (último acesso em 04/08/2009)

SILVA, F. 1994. Mamíferos silvestres: Rio Grande do Sul. 2 ed. Fundação Zoobotânica do Rio Grande do Sul, Porto Alegre, 244 p.

SILVEIRA-NETO, S., NAKANO, O., BARBIN, D. \& NOVA, N.A.V. 1976. Manual de ecologia dos insetos. Editora Agronômica Ceres, São Paulo, 419 p.

TONHASCA Jr., A. 2005. Ecologia e história natural da Mata Atlântica. Interciência, Rio de Janeiro, 197 p.

Universidade de Santa Cruz do Sul - UNISC. 2005. Plano de manejo para a criação da RPPN da UNISC, Sinimbu, RS. UNISC, Santa Cruz do Sul.

WILSON, D.E. \& REEDER, D.M. 2005. Mammal species of the world: a taxonomic and geographic reference. 3 ed. The Johns Hopkins University Press, Baltimore, 2142 p.

Recebido em 18/08/09

Versão reformulada recebida em 29/09/09

Publicado em 11/11/09 